\title{
Non-Identical Particle Femtoscopy in Models with Single Freeze-Out
}

\author{
Adam Kisiel \\ Faculty of Physics, Warsaw University of Technology, ul. Koszykowa 75, 00-662 Warsaw, Poland
}

\author{
Received on 12 December, 2006
}

\begin{abstract}
We present femtoscopic results from hydrodynamics-inspired thermal models with single freeze-out. Nonidentical particle femtoscopy is studied and compared to results of identical particle correlations. Special emphasis is put on shifts between average space-time emission points of non-identical particles of different masses. They are found to be sensitive to both the spatial shift coming from radial flow, as well as average emission time difference coming from the resonance decays. The Therminator Monte-Carlo program was chosen for this study because it realistically models both of these effects. In order to analyze the results we present and test the methodology of non-identical particle correlations.
\end{abstract}

Keywords: Single freeze-out; Femtoscopy; Resonance contribution; Non-identical particle correlations

\section{INTRODUCTION}

The single freeze-out approach [1-3] originates from thermal models of heavy-ion collisions. It is based on thermal fits to particle yields and yield ratios, which are known to work well for RHIC collisions. These ratios are not sensitive to the underlying geometry of the collision, which is what is measured by femtoscopy. The form of the freeze-out geometry must be postulated and should give the overall volume of the system, which is reflected in the absolute yields of particles, as well as the detailed shape of the emission region probed by two-particle correlations. We have postulated such a form of the freeze-out hypersurface which is motivated by hydrodynamics. It has been used to calculate femtoscopic observables, both for identical [4] and non-identical particles. The latter are especially interesting and are the focus of this work. They have been recently measured at SPS and RHIC [5-7]. There have been very few theoretical predictions for these observables $[5,8]$. They contain a crucial and unique piece of information - the difference between the average emission points of two particle types [9-13]. If the particles have different masses, we expect a spatial shift coming from the collective flow of matter. If the particles are of different type, we also expect a very different pattern of emission times, since many particles come from strong decays of resonances. Both of these shifts are interconnected in the measured average emission point difference. Disentangling them is not a trivial task. Single freeze-out models with resonances are perfectly suited for it, as they include realistic modeling of both effects.

\section{FEMTOSCOPY DEFINITIONS}

In this work we will analyze correlation functions between non-identical particles. The femtoscopic correlation function is usually defined as:

$$
C(\mathbf{q}, \mathbf{K})=\frac{P_{2}^{C}(\mathbf{q}, \mathbf{K})}{P_{2}^{0}(\mathbf{q}, \mathbf{K})}
$$

where $P_{2}^{C}$ is the probability to observe two femtoscopically correlated particles at relative momentum q. $P_{2}^{0}$ is the probability where the correlation between particles does not have the femtoscopic component. $\mathbf{K}$ is the average momentum of the pair. In heavy-ion experiments $P_{2}^{C}$ is usually constructed from pairs coming from the same event, while $P_{2}^{0}$ is constructed from pairs where each particle comes from a different event. The events are as close to each other in global characteristics as possible.

In theoretical models one should, in principle, generate particles in such a way that they are already correlated due to their mutual and many-particle interactions. That is however usually computationally not possible. One then makes an assumption that the interaction between particles can be separated from the generation process and we write the most general form of the correlation function that can be used by models:

$$
C(\mathbf{q}, \mathbf{K})=\frac{\int S_{1,2}\left(\mathbf{r}^{*}, \mathbf{q}, \mathbf{K}\right)\left|\Psi\left(\mathbf{q}, \mathbf{r}^{*}\right)\right|^{2} d^{4} \mathbf{r}^{*}}{\int S_{1,2}\left(\mathbf{r}^{*}, \mathbf{q}, \mathbf{K}\right) d^{4} \mathbf{r}^{*}}
$$

where $\mathbf{r}^{*}$ is the pair separation in the pair rest frame (PRF) and $S_{1,2}\left(\mathbf{r}^{*}, \mathbf{q}, \mathbf{K}\right)$ is the pair separation distribution defined as:

$$
\begin{aligned}
S_{1,2}\left(\mathbf{r}^{*}, \mathbf{q}, \mathbf{K}\right)= & \int S_{1}\left(\mathbf{x}_{1}, \mathbf{p}_{1}\right) S_{2}\left(\mathbf{r}^{*}-\mathbf{x}_{2}, \mathbf{p}_{2}\right) \\
& \delta\left(\mathbf{r}^{*}-\mathbf{x}_{1}+\mathbf{x}_{2}\right) d^{4} \mathbf{x}_{1} d^{4} \mathbf{x}_{2}
\end{aligned}
$$

and $S(\mathbf{x}, \mathbf{p})$ is the single-particle emission function provided by the model. For identical particles $S_{1} \equiv S_{2}$ and $S_{1,2}\left(\mathbf{r}^{*}\right)$ is symmetric by definition, for non-identical particles it is not so and $S_{1,2}\left(\mathbf{r}^{*}\right)$ is usually asymmetric. It is an important point which will be discussed later. We also note that the ordering of particles in the pair is important: $S_{1,2}(\mathbf{r}) \equiv S_{2,1}(-\mathbf{r})$. The $\Psi$ function will be discussed in the next chapter.

\section{A. Wave-function of the pair}

In Eq. 2 the amplitude $\Psi$ descibes the change in the probability to detect a pair of particles when they interact with each other. Generally, this (Bethe-Salpeter) amplitude depends on both spatial $\left(\mathbf{r}^{*}\right)$ and time $\left(t^{*}\right)$ separation of the particle emitters in PRF. Usually, it can be approximated by the equal-time $\left(t^{*}=0\right)$ solution $\Psi_{-\mathbf{k}^{*}}\left(\mathbf{r}^{*}\right)$ of the scattering problem viewed in the opposite time direction (hence the $-\mathbf{k}^{*}$ subscript). 
In this work we consider pairs of charged non-identical mesons and baryons. In this case the origin of femtoscopic correlations are Coulomb and strong interactions. However, for pion-kaon and pion-proton systems, as well as for samecharge kaon-proton system, the strong interaction is much weaker than Coulomb interaction at small $k^{*}$. For the opposite charge kaon-proton system, there is a significant strong interaction potential, which is interesting in it's own right, however its detailed study is beyond the scope of this paper. The strong interaction is not essential for our study, so we restrict our study to Coulomb interaction in same-charged pion-kaon, pion-proton and kaon-proton systems. Then:

$$
\left|\Psi\left(\mathbf{q}, \mathbf{r}^{*}\right)^{Q C}\right|^{2}=A_{C}|F(-i \eta, 1, i \xi)|^{2}
$$

where $k^{*}$ is half of pair relative momentum in PRF, $A_{C}$ is the Gamow factor, $F$ is the confluent hypergeometric function, $\eta=1 / k^{*} a_{c}, a_{c}$ is the pair Bohr radius and $\xi=k^{*} r^{*}+\mathbf{k}^{*} \mathbf{r}^{*}$. Please note that the wave-function is calculated in the PRF. $a_{c}$ is $248.5 \mathrm{fm}, 222.6 \mathrm{fm}$ and $83.6 \mathrm{fm}$ for pion-kaon, pion-proton and kaon-proton pair respectively.

\section{NON-IDENTICAL PARTICLE CORRELATIONS}

The correlation between a pair of non-identical particles arises from Coulomb and/or strong interaction. We will concentrate on the Coulomb interaction, but our conclusions hold for strong interactions as well. We will discuss the specifics of the correlations for pairs of unlike particles, emphasizing the differences and similarities to traditional identical particle femtoscopy.

The correlation term (4) is calculated in the pair rest frame and depends on the relative momentum $k^{*}$, relative position $r^{*}$ and the angle $\theta^{*}$ between the two. It needs to be emphasized that the low relative momentum in the pair rest frame, corresponds to close velocities, but not momenta, in the laboratory frame. This is in contrast to identical particle interferometry. This also means that particles from very different momentum ranges are correlated, e.g. pion with velocity 0.7 has a momentum of $0.137 \mathrm{GeV}$, a close-velocity kaon has a momentum of $0.484 \mathrm{GeV}$ and a proton: $0.919 \mathrm{GeV}$. In experiment this often poses a problem, as one needs to have a large momentum acceptance to measure close-velocity pairs.

Looking in detail at the hypergeometric function $F$ from (4):

$$
F=1+r^{*}\left(1+\cos \theta^{*}\right) / a_{C}+\ldots
$$

one notices an important feature of $|\Psi|^{2}$, namely that it is not symmetric with respect to the sign of $\cos \theta^{*}$. For same sign particles $A_{c}$ is less than 1.0 and $F$ is above 1.0 , but since the correlation effect must be negative, $A_{c}|F|^{2}<1$. For a given $k^{*}$ and $r^{*}$ one can have two cases: in one $\cos \theta^{*}<0$, for the other $\cos \theta^{*}>0$. The former will have a larger correlation effect (since $F$ is smaller and cannot overcome $A_{c}$ ) and the latter will have a smaller correlation effect. This asymmetry in the correlation effect can be understood with the help of a simple picture: negative $\cos \theta^{*}$ means that $k^{*}$ and $r^{*}$ are anti-aligned, which means that at first the particles will fly towards each other before they fly away, spending more time close together and thus developing a larger correlation. A positive $\cos \theta^{*}$ means they will fly away immediately, having no time to interact. This asymmetry is an intrinsic property of the Coulomb interaction and is also present for identical particles. However in that case the wave-function symmetrization requires one to add a second term to (4) which has the same asymmetry with the opposite sign, so that the overall asymmetry is zero, as it must be.

If we were somehow able to divide pairs in two groups one in which $\left\langle\cos \theta^{*}\right\rangle>0$ and the other in which $\left\langle\cos \theta^{*}\right\rangle<0$ one would obtain two different correlation functions, out of which the latter would show a stronger correlation effect. Obviously we cannot select pairs based on the $\theta^{*}$ angle, as it is not measured. However we do have one angle on which we can select - the angle $\Psi$ between pair velocity $v$ and pair relative momentum $k^{*}$. In the transverse plane $\cos \Psi>0$ means that $k_{\text {out }}^{*}>0$. We also notice that in the transverse plane

$$
\Psi=\theta^{*}+\phi,
$$

where $\phi$ is the angle between the pair velocity $v$ and the relative position $r^{*}$. This angle is not measured as accurately. One can also show that when one averages over all possible positions of $r^{*}$ one can write [14]

$$
\operatorname{sign}\langle\cos \Psi\rangle=\operatorname{sign}\left\langle\cos \theta^{*}\right\rangle \operatorname{sign}\langle\cos \phi\rangle \text {. }
$$

One can then propose a measurement: to divide pairs into two groups, one with $k_{\text {out }}^{*}>0 \equiv \cos \Psi>0$ and the other with $k_{\text {out }}^{*}<0 \equiv \cos \Psi<0$. Then one constructs two correlation functions $C_{+}$and $C_{-}$from the two groups. If one observes that $\left|C_{+}-1\right| /\left|C_{-}-1.0\right|>1.0$ it can only happen if $\langle\cos \phi\rangle<0$ as can be seen from Eq. (7). In other words this can happen only if the average emission points of two particle species are, on the average, separated in the direction of the pair velocity, and this separation is anti-aligned with the velocity. By the same reasoning, if $\left|C_{+}-1\right| /\left|C_{-}-1.0\right|<1.0$ this separation is aligned with the pair velocity. Let us restate the conclusion of this paragraph: using the fact that the correlation effect is asymmetric with respect to the sign of $\cos \left(\theta^{*}\right)$ and the measured angle $\Psi$, we can tell whether an average emission position of two different particle species is the same or not, and if it is not, is the difference is the same or opposite to the direction of pair velocity $v$. More quantitative analysis shows, that the double ratio $C_{+} / C_{-}$is also monotonously dependent on the value of this shift between particles, so the magnitude of the shift can be inferred from it. This is a unique feature of non-identical particle correlations, as such information cannot be obtained from any other measurement.

A simple formula can be written for the behavior of the double-ratio at low $k^{*}[11]$ :

$$
\lim _{k^{*} \rightarrow 0} C_{+} / C_{-}=1+2\left\langle r_{i}^{*}\right\rangle / a_{C}
$$

which also illustrates the sensitivity of the double ratio to the mean shift between emission points. It is however difficult to use this formula in the experiment, as the low $k^{*}$ region 
usually suffers from low statistics and two-track separation problems.

The above consideration has been performed for the pair rest frame. Experimentally, however, one would like to learn something about the source itself, which requires the knowledge of the source in its rest frame. In symmetrical collisions in collider experiments (which is what we will consider later in the manuscript) we can assume that the source frame coincides with the laboratory frame. One can write a simple formula for the relative separation in pair rest frame $r^{*}$ as a function of the pair separation in the source frame $r$ :

$$
\begin{aligned}
r_{\text {out }}^{*} & =\gamma_{t}\left(r_{\text {out }}-\beta_{t} \Delta t_{L}\right) \\
r_{\text {side }}^{*} & =r_{\text {side }} \\
r_{\text {long }}^{*} & =\gamma_{l}\left(r_{\text {long }}-\beta_{l} \Delta t\right) \\
\Delta t_{L} & =\gamma_{l}\left(\Delta t-\beta_{l} r_{\text {long }}\right)
\end{aligned}
$$

where the long direction is defined as the one parallel to the velocity of the colliding nuclei, out as parallel to the pair velocity in the transverse direction, and side as perpendicular to the other two. The pair velocities are $\beta_{l}=p_{l} / E$, $\gamma_{l}=1 / \sqrt{1-\beta_{l}^{2}}, \beta_{t}=p_{\perp} / m_{\perp}, \gamma_{t}=1 / \sqrt{1-\beta_{t}^{2}}$. One can see that the average shift in $r_{\text {out }}^{*}$ may mean non-zero average spatial shift $r_{\text {out }}$, non-zero average emission time difference $\Delta t$ or a combination of the two.

\section{THERMINATOR}

The Therminator program [15] is the numerical implementation of the single freeze-out model [1-3]. It includes all particles listed by the Particle Data Group [16]. We use the version of the model based on the blast-wave type parametrization, which is hydrodynamics-inspired [8]. Therefore our model includes the effects of radial flow, which is apparent, e.g., in the $m_{T}$ dependence of the pion "HBT radii" [4]. This is an important feature, as the space-momentum correlation coming from radial flow is one of the origins of emission asymmetries between various particle species. The emission function is changed slightly from the version described in [15]. A quasi-linear velocity profile as a function of $\rho$ is added. The freeze-out hypersurface is then defined as:

$$
\tilde{\tau}=\tau=\text { const }, \quad v_{r}=\tanh \alpha_{\perp}=\frac{\rho / \rho_{\max }}{v_{T}+\rho / \rho_{\max }}
$$

where $\tau, \rho_{\max }$ and $v_{T}$ are parameters of the model. The quasilinear velocity profile has the desired features - it is zero for $\rho=0$, is almost linear for reasonable values of $v_{T}$, and cannot go higher than 1.0. The emission function is then:

$$
\begin{gathered}
\frac{d N}{d y d \varphi p_{\perp} d p_{\perp} d \alpha_{\|} d \phi \rho d \rho}=\frac{\tau}{(2 \pi)^{3}} m_{\perp} \cosh \left(\alpha_{\|}-y\right) \times \quad(11) \\
\left\{\exp \left[\beta \frac{m_{\perp} \cosh \left(\alpha_{\|}-y\right)-p_{\perp} v_{r} \cos (\varphi-\phi)}{\sqrt{1-v_{r}^{2}}}-\beta \mu\right] \pm 1\right\},
\end{gathered}
$$

where $(\rho, \phi)$ is the transverse emission point in radial coordinates, $\alpha_{\|}$is the space-time rapidity of the emission point, $y$ is the particle rapidity, $\left(p_{T}, \varphi\right)$ is the particle's transverse momentum in radial coordinates, and $m_{T}$ is its transverse mass. The inverse temperature $\beta$ and $\mu$, the particle-specific chemical potentials, are also parameters of the model.

The fit to STAR Collaboration data [17] has been performed with this model and the following values of the parameters were found to best reproduce the observed pion and kaon spectra in central AuAu collisions: $\tau=8.55 \mathrm{fm}, \rho_{\max }=8.92$ $\mathrm{fm}, a=-0.5, v_{T}=1.41$. The negative value of the $a$ parameter means that the freeze-out hypersurface has a negative slope (an anti-correlation) in the $\rho-t$ plane, or in other words the freeze-out occurs outside-in [4]. The thermodynamic parameters $T$ and $\mu$ were the same as in [4]. The velocity profile for these parameters is shown in Fig. 1. The average velocity is 0.31 .

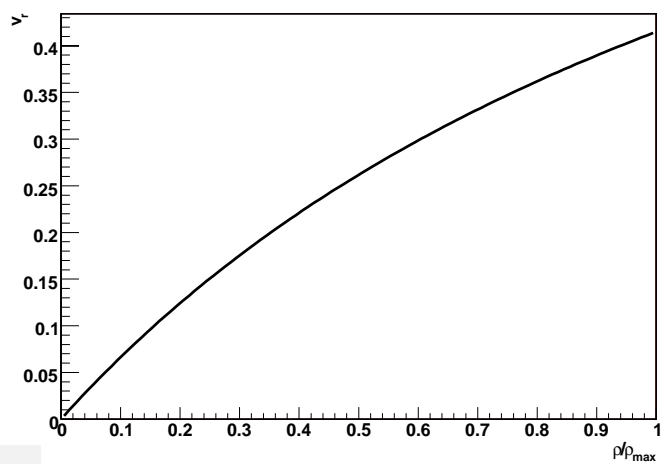

FIG. 1: Radial velocity profile for the parameters used in this study.

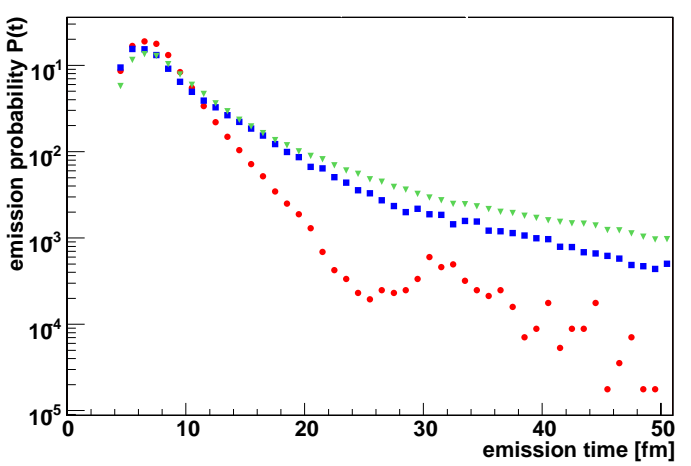

FIG. 2: (Color online) Probability to emit a pion (green triangles), kaon (blue squares) and proton (red circles) as a function of time.

TABLE I: Average emission times from Therminator

\begin{tabular}{|l|l|}
\hline Particle species & Average emission time \\
\hline \hline pion & $12.3 \mathrm{fm} / \mathrm{c}$ \\
\hline kaon & $10.7 \mathrm{fm} / \mathrm{c}$ \\
\hline proton & $7.9 \mathrm{fm} / \mathrm{c}$ \\
\hline
\end{tabular}


The simulation proceeds as follows. First, using a MonteCarlo integration procedure as a particle generator, all particles (stable and unstable) are generated according to the emission function (11). Each particle is given an emission point on the freeze-out hypersurface and a momentum. Then, all unstable particles decay after some random time dependent on their width. They propagate to the decay point, and this point is then taken as the space-time origin of the daughter particles. Two- and three-particle decays are implemented. The process is repeated for cascade decays, until only stable particles remain. While each particle has its emission point either on the freeze-out hypersurface (we call such particles primordial) or at the decay point of the heavier resonance, the full history of decays can be reconstructed from the output files. This treatment of resonance propagation and decay is of crucial importance for non-identical particle correlations. It introduces delays in emission time, which are different for different particle species. It depends on the number of resonances that decay into the particle of interest, their widths and velocities. An illustration can be seen on Fig. 2. The Monte-Carlo procedure we used is the most efficient way of studying it, more precise results can only be obtained by using a full-fledged hadronic rescattering model. In our work hadronic rescattering is not taken into account, which is one of the simplifications assumed in the single freeze-out model.

\section{A. Calculating the correlation function}

It is possible to use Eq. 2 to obtain the model correlation function. The integral is calculated numerically. One takes particles generated by Therminator. Then one combines them into pairs and creates two histograms - in one of them one stores the $|\Psi|^{2}$ of the pair (4), in the other - unity for each pair. The result of the division of the two histograms is the average correlation effect in each bin, which is the correlation function per Eq. (2). This is the so-called "two-particle weight" method of calculating the correlation function from models. It is the only one which takes into account the two-particle Coulomb interaction exactly, a feature which is necessary for our study. In this procedure each pair is treated separately, as one goes to the pair rest frame (different for each pair) as the calculation of the $\Psi$ is done most naturally in this system.

\section{ASYMMETRY ANALYSIS}

In section III it was shown that non-identical particle correlations are sensitive to the shifts between average emission points of different particle species. However, we have not discussed if and how such asymmetries could arise. In Fig. 3 the average emission points of pions, kaons and protons from THERMINATOR where shown for pairs which have similar velocity, pointing horizontally to the right. One sees that the average emission points of pions, kaons and protons is not the same in the out direction, while it is 0 for the side direction for all of them. One can also see that the average size of the emission region is decreasing with particle mass, a known effect of
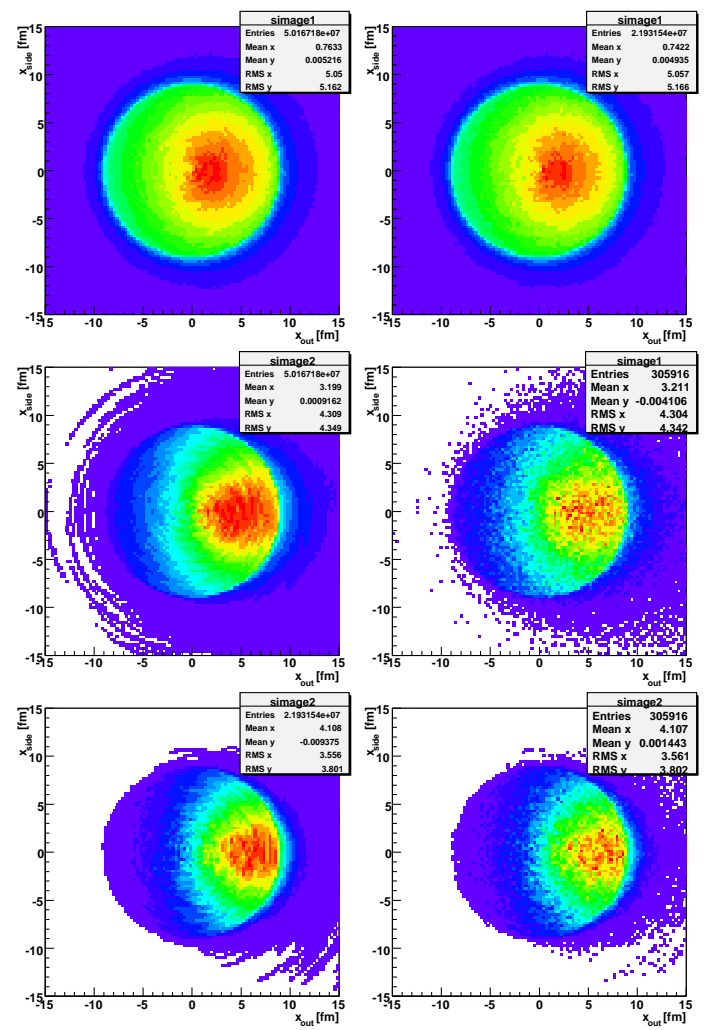

FIG. 3: (Color online) Distribution of emission points of pions (upper plots), kaons (center plots) and protons (lower plots) versus pairwise out and side directions. Left and right side are emission points of the same particle from different system, but for the same pair velocity range (for pions: left $-\pi K$, right $-\pi p$, for kaons: left $-\pi K$, right $-K p$, for protons: left $-\pi p$, right $-K p$.

radial flow, usually referred to as " $m_{T}$ scaling" of HBT radii. The shift that we observe is also a direct but distinctly different consequence of radial flow present in our simulation. The $m_{t}$ dependence of the average emission point has been discussed in detail in [5] and the formula for the longitudinal boost-invariant hydrodynamic model (similar to the one used in this work) has been given:

$$
\langle x\rangle=r_{0} \frac{\beta_{0} \beta_{t}}{\beta_{0}^{2}+T / m_{t}}
$$

where $r_{0}$ is the radius of the system, $\beta_{t}$ is the transverse velocity of the particle and $\beta_{0}$ is the parameter of the model characterizing the strength of flow. One can understand it by the following argument. Pions and protons of the same velocity have very different momenta. By inspecting Eq. (11) one observes that the correlation between the space-momentum emission point direction $\phi$ and the momentum direction $\varphi$ is controlled by the factor: $\exp \left(\beta p_{\perp} \cos (\varphi-\phi)\right)$. So the higher the momentum, the stronger the correlation. The temperature $1 / \beta$ is of course identical for both particle species. Therefore, in a close-velocity pair, the proton emission direction will be much more correlated with its momentum direction. And the momentum, due to radial flow, is always pointing "outward", 
so the emission points will tend to be concentrated near the edge of the system, in the direction of emission. For pions on the other hand, there is almost no correlation between the two directions, so they are emitted from the whole source. The effect is clearly seen in the figure, as a difference between mean emission points in the "out" direction:

$$
r_{\text {out }}^{\pi p}=x_{\text {out }}^{\pi}-x_{\text {out }}^{p}
$$

This is the spatial shift between particles in the source frame. Measuring it is the main goal of non-identical particle femtoscopy. Observing such a shift in the experiment would be direct evidence of the collective behavior of matter, which is one of the necessary conditions to claim the discovery of the quark-gluon plasma.

One must remember that the asymmetries measured in the correlation function are averaged over the source. Therefore, the connection between a shift in the laboratory frame and in the pair rest frame is:

$$
\left\langle r_{\text {out }}^{*}\right\rangle=\left\langle\gamma\left(r_{\text {out }}-\beta \Delta t\right)\right\rangle
$$

TABLE II: Average space shifts from Therminator

\begin{tabular}{|l|l|l|l|}
\hline$\beta_{t}$ of the pair & $r_{\pi K}$ & $r_{\pi p}$ & $r_{K p}$ \\
\hline \hline $0.35-0.5$ & $-1.9 \mathrm{fm}$ & $-2.5 \mathrm{fm}$ & $-0.6 \mathrm{fm}$ \\
\hline $0.5-0.65$ & $-2.4 \mathrm{fm}$ & $-3.4 \mathrm{fm}$ & $-0.9 \mathrm{fm}$ \\
\hline $0.65-0.8$ & $-2.9 \mathrm{fm}$ & $-3.9 \mathrm{fm}$ & $-1.1 \mathrm{fm}$ \\
\hline $0.8-0.95$ & $-3.0 \mathrm{fm}$ & $-4.3 \mathrm{fm}$ & $-1.2 \mathrm{fm}$ \\
\hline
\end{tabular}

TABLE III: Average time shifts from Therminator

\begin{tabular}{|l|l|l|l|}
\hline$\beta_{t}$ of the pair & $\Delta t_{\pi K}$ & $\Delta t_{\pi p}$ & $\Delta t_{K p}$ \\
\hline \hline $0.35-0.5$ & $3.8 \mathrm{fm} / \mathrm{c}$ & $6.2 \mathrm{fm} / \mathrm{c}$ & $2.3 \mathrm{fm} / \mathrm{c}$ \\
\hline $0.5-0.65$ & $3.6 \mathrm{fm} / \mathrm{c}$ & $5.7 \mathrm{fm} / \mathrm{c}$ & $2.1 \mathrm{fm} / \mathrm{c}$ \\
\hline $0.65-0.8$ & $3.2 \mathrm{fm} / \mathrm{c}$ & $5.1 \mathrm{fm} / \mathrm{c}$ & $1.9 \mathrm{fm} / \mathrm{c}$ \\
\hline $0.8-0.95$ & $2.6 \mathrm{fm} / \mathrm{c}$ & $4.2 \mathrm{fm} / \mathrm{c}$ & $1.5 \mathrm{fm} / \mathrm{c}$ \\
\hline
\end{tabular}

From Eq. (9) one sees that the measurable shift $r_{\text {out }}^{*}$ is a combination of the spatial shift $r_{\text {out }}$, and the emission time shift $\Delta t$. The former effect is of special interest and has been studied in detail in [8]. However, the effect of time difference has not been adequately studied so far. The Therminator model has been chosen to perform this task, because it includes both effects in the same calculation in a self-consistent way. Fig. 2 shows the probability to emit a pion, a kaon or a proton at a given time. One sees that the average emission times of this particle species, listed in Tab. I, are different in the laboratory frame. This will affect the observed asymmetries.

In Fig. 4 one can see the components of the emission asymmetries in the laboratory frame, obtained directly from the emission functions $S_{1,2}$. The time and space components for all considered systems are compared. They are of the same order for all systems. The overall asymmetry is the combination of the two.

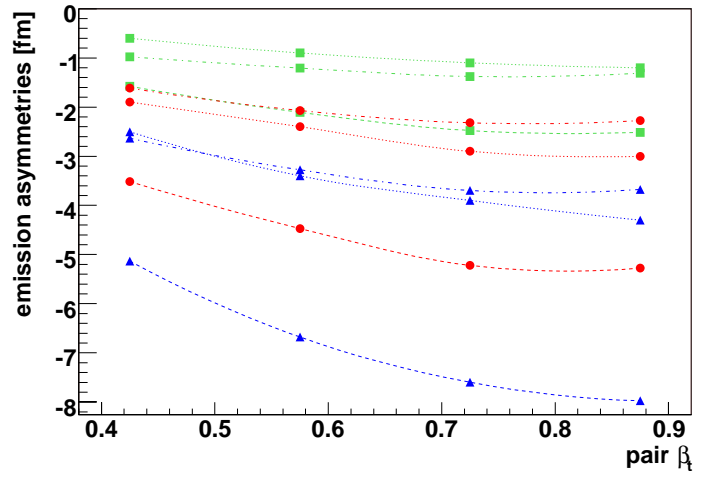

FIG. 4: (Color online) Components of the average shift between particle species in the laboratory frame. The dotted line is the space component, the dash-dotted line is the time component, and the dashed line is the full asymmetry. Red circles are for the $\pi K$ system, blue triangles are for $\pi p$, green squares are for $K p$.

\section{A. Obtaining femtoscopic information}

The correlation function for non-identical particles is given by Eq. (2). In order to obtain femtoscopic information from the experimental correlation function one needs to perform a fit procedure. In traditional HBT measurement the integral analog to Eq. (2) can be performed analytically to obtain a simple fit function. It is not possible for the general case of non-identical particles, where the following procedure must be applied. Usually it is assumed that the emission function factorizes into space and momentum components. A form of the spatial emission function is then postulated:

$$
S(\mathbf{r}) \approx \exp \left(-\frac{\left(r_{\text {out }}-\mu_{\text {out }}\right)^{2}+r_{\text {side }}^{2}+r_{\text {long }}^{2}}{2 R^{2}}\right)
$$

defined in the laboratory frame. Then, the momenta of pairs of particles are taken from experiment. Their emission points are randomly generated according to (15), with some assumed values of source parameters: the gaussian source radius $R$ and the shift in the outwards direction $\mu_{\text {out }}$. For each pair the weight is then calculated according to (4), and the average of the weights over all pairs as a function of $k^{*}$ is constructed. This is a theoretical correlation function according to (2). This function can be compared via a $\chi^{2}$ test to the experimental correlation function being fitted. By varying parameters $R$ and $\mu_{\text {out }}$ a function can be found which best describes the input one. Parameters of the source which produce this function are taken as the best-fit values. In this way femtoscopic information is obtained from the non-identical particle correlation function. In this work the model correlation functions calculated according to Eq. 2 were treated as "pseudoexperimental" ones. A dedicated program $[14,18]$ has been used to perform a fit on them in a manner as closely resembling the experimental situation as possible. It was a direct test of the methodology of non-identical particle correlations. 


\section{B. Sum rule for shifts between different particle species}

If we have three different particle species, we have three average emission point shifts that we can measure: $\mu^{\pi K}, \mu^{\pi p}$ and $\mu^{K p}$. However, if we take the same group of e.g. pions for $\pi-K$ correlations and $\pi-p$ correlations (and similarly the same group of kaons and protons), we might expect that a simple sum rule holds:

$$
\mu^{\pi p}=\mu^{\pi K}+\mu^{K p}
$$

and only two of the shifts are independent.

Non-identical particles are correlated if they have close velocities. If we select pairs of particles with some pair velocity, we expect that the correlated particles themselves also have velocities in this range. Therefore if we compare pions that form the $\pi-K$ correlation in the pair $\beta_{t}$ range between 0.5 and 0.65 , and pions from the $\pi-p$ correlation in the same pair $\beta_{t}$ range we may assume that these are the same pions (providing that we construct the correlation functions from the same events). That shows that pair $\beta_{t}$ is the correct variable to select on, when studying momentum dependence of the femtoscopic parameters from non-identical particle correlations. It also shows that we should indeed expect the sum rule (16) to hold separately in each $\beta_{t}$ bin.

\section{Two-particle versus single particle sizes}

The size of the two-particle source is a combination of the individual single-particle source sizes:

$$
\begin{aligned}
\sigma_{\pi K} & =\sqrt{\sigma_{\pi}^{2}+\sigma_{K}^{2}} \\
\sigma_{\pi p} & =\sqrt{\sigma_{\pi}^{2}+\sigma_{p}^{2}} \\
\sigma_{K p} & =\sqrt{\sigma_{K}^{2}+\sigma_{p}^{2}}
\end{aligned}
$$

where $\sigma$ is a width of a gaussian fitted to the corresponding emission function (either single- or two-particle). One can immediately see that the sizes are not independent, similar to the shifts. One can also use the combination of the measured two-particle source sizes to obtain the single particle sizes:

$$
\begin{aligned}
\sigma_{\pi} & =\sqrt{\frac{\sigma_{\pi K}^{2}+\sigma_{\pi p}^{2}-\sigma_{K p}^{2}}{2}} \\
\sigma_{K} & =\sqrt{\frac{\sigma_{\pi K}^{2}-\sigma_{\pi p}^{2}+\sigma_{K p}^{2}}{2}} \\
\sigma_{p} & =\sqrt{\frac{-\sigma_{\pi K}^{2}+\sigma_{\pi p}^{2}+\sigma_{K p}^{2}}{2}}
\end{aligned}
$$

They can then be compared to single-particle source sizes obtained from regular identical particle interferometry to see whether the size of the system is described self-consistently.

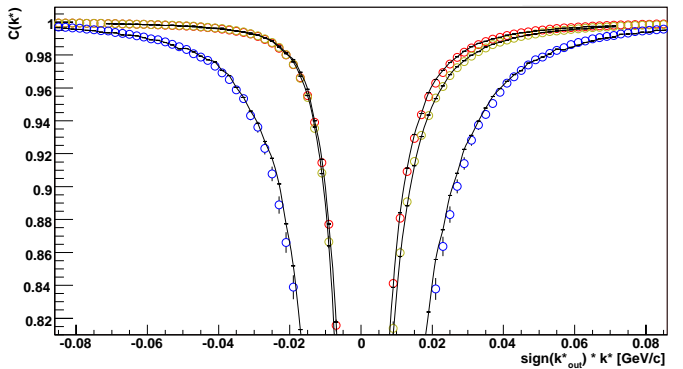

FIG. 5: (Color online) Correlation functions for pion-kaon (red), pion-proton (yellow) and kaon-proton (blue) for pairs with velocity between 0.5 and 0.65 . The lines are fits to the correlation function.

\section{RESULTS AND DISCUSSION}

The analysis of the correlation functions for pion-kaon, pion-proton and kaon-proton systems has been performed based on the Therminator model and the two particle weight method, described in the previous paragraphs. Examples of the obtained correlation functions are shown in Fig. 5. As expected for like-sign pairs they go below unity at low $k^{*}$. The correlation effect is the smallest for pion-kaon and largest for kaon-proton due to the difference in the Bohr radii of the pairs. It is a fortunate coincidence, as for kaon-proton one expects to have the smallest statistics in the experiment, but due to a large correlation effect the measurement should be doable for a data sample similar to the one in which pion-kaon measurement is possible.

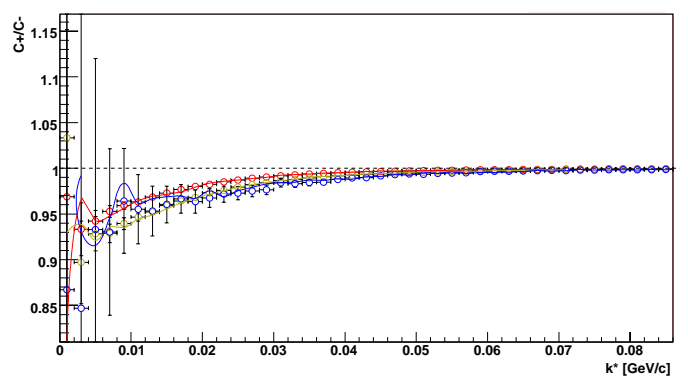

FIG. 6: (Color online) Double ratios for pion-kaon (red), pionproton (yellow) and kaon-proton (blue) for pairs with velocity between 0.5 and 0.65 . The lines are double ratios calculated from the fits to the correlation function.

In Fig. 6 examples of the asymmetry measurement - the double ratios $C_{+} / C_{-}$for pion-kaon, pion-proton and kaonproton systems, are shown. One can see a significant signal, which indicates that the correlation functions are indeed sensitive to the asymmetries described in the previous paragraph. In our analysis we have adopted a convention in which a lighter particle is always taken as first in the pair. All the double ratios go below unity, which means that, on average, particles which are lighter are emitted closer to the center of the system or later (or both). This is exactly consistent with the qualitative picture of spatial shifts coming from radial flow 
shown in Fig. 3, and with emission time differences coming from resonance decays, shown in Fig. 2 and Tab. III.

The correlation functions have been fitted using the numerical Monte-Carlo procedure described in Sect. IV. Fig. 5 shows the resulting best-fit functions as solid lines. The fit is performed simultaneously to the positive $k_{\text {out }}^{*}$ and negative $k_{\text {out }}^{*}$ part of the correlation function. The lines in Fig. 6 are simply the two parts of the fit function from Fig. 5 divided by each other, they are not fit independently.

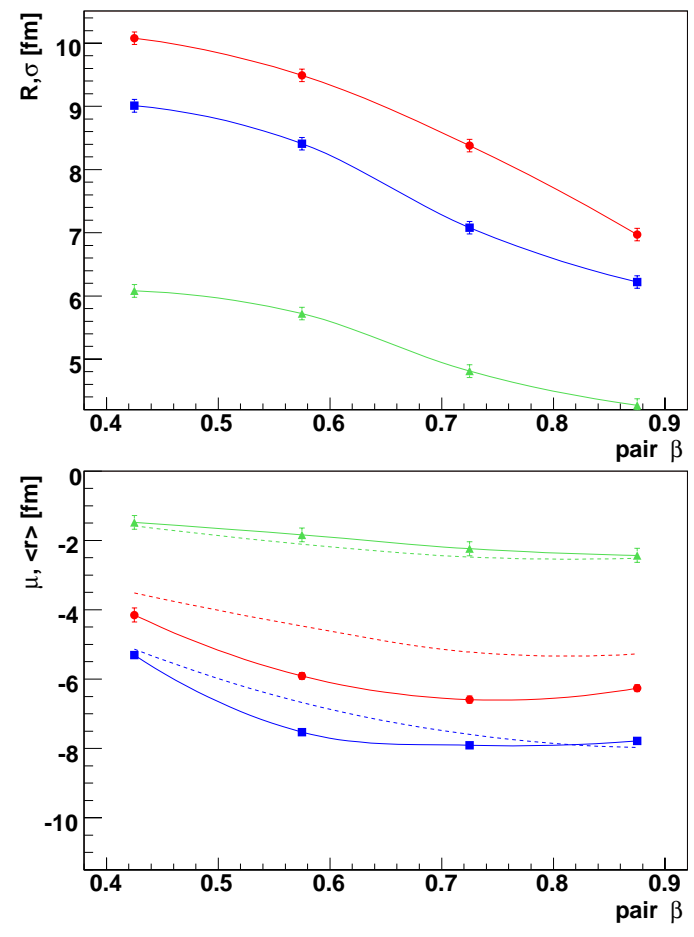

FIG. 7: (Color online) Parameters of the non-identical particle sources from fits. In the upper panel source size is shown, in the lower - shift between mean emission points in the direction of pair transverse momentum. Red circles are for pion-kaon, blue squares for pion-proton, green triangles for kaon-proton. Dashed lines are input overall asymmetries from Fig. 4.

The results of the fit are shown in Fig. 7. We find that the size of the emitting system decreases with pair velocity for all considered pair types. This is consistent with the " $m_{T}$ scaling" of the HBT radii observed in the identical particle femtoscopy calculations. The shifts between various particle species have an expected ordering - the larger the mass difference, the larger the shift. This means that for all observed systems the lighter particle is emitted, on the average, closer to the center and/or later than the heavier one. It also means that time differences do not change the qualitative behavior of the observed asymmetries. However, consulting Tab. III and Fig. 4, one can see that they do contribute to the observed asymmetry. The dashed lines in Fig. 7 show the overall asymmetry from Fig. 4 as predicted directly from the emission functions $S_{1,2}(\mathbf{r})$ from the model. The agreement in absolute values and in the general trends between them and the final fit values is within $1.0 \mathrm{fm}$.
This is of crucial importance for the experiment. It means that by performing an advanced fit of all three combinations of non-identical particle correlation functions one can indeed infer the properties of the underlying two-particle emission functions, and therefore obtain a new, unique piece of information about the dynamics of the collision.

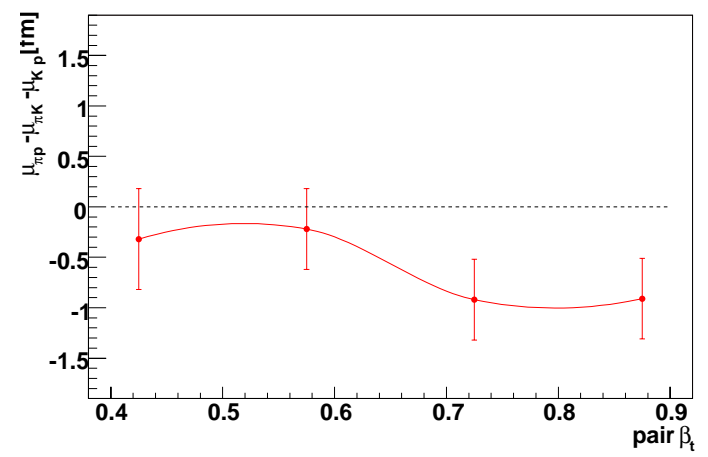

FIG. 8: (Color online) Test of the sum rule of mean shifts between various particle types.

One can also test the validity of the sum rule (16). The test using the fit values from Fig. 7 is shown in Fig. 8. One can see that the rule is valid for smaller pair velocity and holds only approximately for larger velocities. This should be compared to results in Tab. II, where the shifts obtained from the separation distributions themselves follow the sum rule with the accuracy of $0.1 \mathrm{fm}$. Also, one can expect deviations on the order of the differences between the input and fitted values of the shift shown in Fig. 7. One can see that within these systematic limits the agreement is acceptable. This is another important conclusion for the experiment. If shifts between all three combinations are measured in the comparable pair velocity window one expects the sum rule for the shifts (16) to hold. It can be used as a quality check on the data.

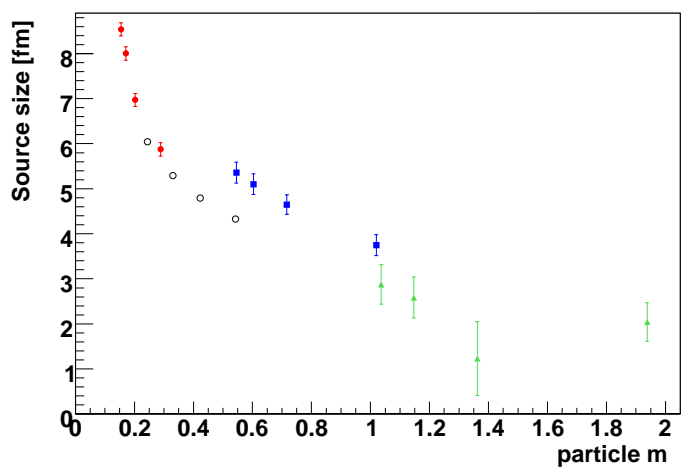

FIG. 9: (Color online) Single particle source sizes obtained from non-identical particle correlation fits, according to (18). Red circles are pions sizes, blue squares - kaon, green triangles - proton. Open circles are sizes obtained from identical-particle $\pi$ correlations.

One can also use the fitted two-particle radii for all systems to obtain the single particle radii according to (18). The results are shown in Fig. 9. The radii are reasonable and exhibit 
the expected " $m_{T}$ scaling" for all particle species. Therefore an experiment can extract the information not only about the asymmetries of emission but also about the size of the system. The comparison between the size estimates obtained from non-identical particle correlations and "HBT radii" obtained from identical pion interferometry was done. Both sizes for pions are consistent with each other. One must remember that in the case of Therminator model, the obtained source functions exhibit large long-range non-gaussian tails [4]. On the other hand, both identical and non-identical femtoscopic sizes were obtained assuming a perfect gaussian source. Both of these measures can be sensitive to long-range tails in a different way, so the comparison must be done with caution.

\section{SUMMARY}

We have presented the first complete set of calculations of non-identical particle correlations from the single freeze-out models with complete treatment of resonances. Non-identical particle femtoscopy method was shown to be sensitive to both the size and emission asymmetries in the system. The observed effects have been shown to be under control both qualitatively and quantitatively. The method to extract femtoscopic information from such correlation functions have been presented and employed to the "pseudo-experimental" functions obtained from the model. The results of the fit have been shown to be in agreement with the characteristics of the input source. Several consistency checks on the experimental data have been proposed and their validity tested. A method to test the consistency of identical and non-identical femtoscopy results has also been proposed.

The femtoscopic analysis of non-identical particle correlations has shown that the emission asymmetries between pions, kaons and protons are expected to occur in heavy-ion collisions. Spatial asymmetry coming from radial flow was observed. Time asymmetry coming from resonance propagation and decay was also estimated and found to be on the order of the space asymmetry in the laboratory frame and in the same direction for all tested systems. Therefore a realistic and selfconsistent estimate of the two effects has been given for the first time. Consistency between identical and non-identical femtoscopic sizes was tested and found to be within $1.0 \mathrm{fm}$.

Predictions for both the size of the system as well as emission asymmetry have been given for central AuAu collisions for pion-kaon, pion-proton and kaon-proton correlations.

\section{Acknowledgements}

This work was supported by Polish Ministry of Science and Higher Education, grants no. 0395/P03/2005/29 and 134/E365/SPB/CERN/P-03/DWM 97/2004-2007. I would like to thank Prof. W. Broniowski and Prof. W. Florkowski for very fruitful discussions.
[1] W. Florkowski, W. Broniowski, and M. Michalec, Acta Phys. Polon. B 33, 761 (2002) [arXiv:nucl-th/0106009].

[2] W. Broniowski and W. Florkowski, Phys. Rev. Lett. 87, 272302 (2001) [arXiv:nucl-th/0106050].

[3] W. Broniowski, A. Baran, and W. Florkowski, AIP Conf. Proc. 660, 185 (2003) [arXiv:nucl-th/0212053].

[4] A. Kisiel, W. Florkowski, and W. Broniowski, Phys. Rev. C 73, 064902 (2006) [arXiv:nucl-th/0602039].

[5] R. Lednicky, Proc. CIPPQG'01, Palaiseau, France, Phys. Atom. Nucl. 67, 72 (2004) [arXiv:nucl-th/0112011].

[6] C. Blume et al., Nucl. Phys. A 715, 55 (2003) [arXiv:nuclex/0208020].

[7] J. Adams et al. [STAR Collaboration], Phys. Rev. Lett. 91, 262302 (2003) [arXiv:nucl-ex/0307025].

[8] F. Retiere and M. A. Lisa, Phys. Rev. C 70, 044907 (2004) [arXiv:nucl-th/0312024].

[9] R. Lednicky, V.L. Lyuboshitz, Yad. Fiz. 35, 1316 (1982) (Sov. J. Nucl. Phys. 35, 770 (1982); Proc. Int. Workshop on Paricle Correlations and Interferometry in Nuclear Collisions, CORINNE 90, Nantes, France 1990 (ed. D. Ardouin, World Scientific 1990) p. 42; Heavy Ion Physics 3, 1 (1996).

[10] R. Lednicky, V. L. Lyuboshits, B. Erazmus, and D. Nouais,
Phys. Lett. B 373, 30 (1996).

[11] S. Voloshin, R. Lednicky, S. Panitkin, and N. Xu, Phys. Rev. Lett. 79, 4766 (1997) [arXiv:nucl-th/9708044].

[12] R. Lednicky, "Finite-size effects on two-particle production in continuous and discrete spectrum", arXiv:nucl-th/0501065.

[13] A. Kisiel, "Non-identical particle femtoscopy in heavy-ion collisions," AIP Conf. Proc. 828, 603 (2006).

[14] A. Kisiel, "Studies of non-identical meson-meson correlation at low relative velocities in relativistic heavy-ion collisions registered in the STAR experiment", PhD Thesis, Warsaw Univeristy of Technology (2004).

[15] A. Kisiel, T. Taluc, W. Broniowski, and W. Florkowski, Comput. Phys. Commun. 174, 669 (2006) [arXiv:nucl-th/0504047].

[16] S. Eidelman et al. [Particle Data Group], "Review of particle physics," Phys. Lett. B 592, 1 (2004).

[17] J. Adams et al. [STAR Collaboration], Phys. Lett. B 616, 8 (2005) [arXiv:nucl-ex/0309012].

[18] A. Kisiel, "CorrFit - a program to fit arbitrary two-particle correlation functions", NUKLEONIKA 2004, 49 (Suppl. 2), s81 (2004). 